\title{
Advanced Urothelial Carcinoma
}

National Cancer Institute

\section{Source}

National Cancer Institute. Advanced Urothelial Carcinoma. NCI Thesaurus. Code C148493.

Urothelial carcinoma that has spread extensively to other anatomic sites or is no longer responding to treatment. 